\title{
Relación entre el riesgo nutricional y el número de interconsultas realizadas al servicio de nutrición clínica según el servicio hospitalario
}

\author{
Relation between nutritional risk and interconsultation made to the clinical \\ nutrition service according to hospital service
}

\author{
Erika Areli Rosas Gonzalez', Karolina Álvarez Altamirano², Mónica Patricia Bejarano Rosales², \\ Vanessa Fuchs Tarlovsky²
}

Recibido: 17 de Julio de 2018. Aceptado para publicación: 15 de Agosto de 2018 https://doi.org/10.35454/rncm.v1n2.043

\section{Resumen}

Antecedentes: la nutrición clínica es una disciplina que ha tenido gran impacto en los últimos años. Sin embargo, en el ámbito hospitalario es necesario que los médicos tratantes hagan un seguimiento más minucioso a los pacientes con riesgo nutricional con el fin de ofrecer el tratamiento nutricional oportuno.

Objetivo: evaluar la proporción de interconsultas al servicio de nutrición clínica comparado con el porcentaje de riesgo nutricional de cada servicio.

Metodología: se realizó un estudio observacional, analítico, transversal en los servicios del Hospital General de México, en el cual se comparó el número de pacientes diagnosticados con riesgo nutricional, evaluado mediante la herramienta NRS-2002 y el número de interconsultas que se recibieron por servicio hospitalario durante un año. Los datos recolectados de los registros de cada área fueron valorados en forma de proporciones por medio del programa STATA V. 14.

Resultados: se analizaron en total 31.225 pacientes, de los cuales el $19,20 \%$ presentó riesgo nutricional. A $81,42 \%$ de los pacientes con riesgo nutricional les solicitaron interconsulta al servicio de nutrición clínica. Los servicios con mayor porcentaje de riesgo nutricional fueron nefrología $(95,26 \%)$, geriatría $(66,46 \%)$ y oncología (63,23\%). Los servicios con menor número de interconsultas fueron oftalmología (1), urología (91), nefrología (67) y geriatría (70) a pesar de tener un porcentaje importante de pacientes con riesgo nutricional.

Conclusión: el porcentaje de solicitud de interconsulta al servicio de nutrición clínica por parte del médico tratante no se relaciona con el porcentaje de riesgo nutricional por servicio. Se hace necesario generar conciencia sobre el seguimiento nutricional a los pacientes hospitalizados.

Palabras clave: riesgo nutricional, tamizaje nutricional, terapia nutricional, NRS- 2002.

1 Escuela Superior de Medicina IPN, Maestría en Ciencias de la Salud,

\section{Summary}

Background: Clinical nutrition is a discipline which has had a big impact in previous years. However, in a hospital level it is still necessary that treating doctors give a more thorough follow up to patients with nutritional risk to enable timely nutritional treatment. The objective of this study was to evaluate the proportion of interconsultation to the clinical nutrition service compared with the percentage of nutritional risk of each service.

Methodology: An observational cross sectional study was performed in Mexico General Hospital in which the number of patients diagnosed with nutritional risk evaluated with NSR2002 was compared with the number of inter consultations received through hospital service during a year. The data collected from the files of each area were evaluated in proportion forms through STATA V. 14 program.

Results: A total of 31.225 patients were analyzed out of which $19.20 \%$ evidenced nutritional risk. An interconsultation to the clinical nutrition service was requested for $81.42 \%$ of the patients with nutritional risk. The services with a higher percentage of nutritional risk were Nephrology (95.26\%), geriatric (66.46\%) and oncology (63.23\%). The services with fewer number of inter consultations were ophthalmology (1), urology (91), nephrology (67) and geriatric (70) despite not having an important percentage of patients with nutritional risk.

Conclusion: The percentage of consultation requirement to the clinical nutrition service by the treating doctor is not related with the percentage of nutritional risk in each service. It is necessary to generate consciousness on the nutritional follow up of hospitalized patients.

Keywords: Nutritional risk; Nutritional triage; Nutritional therapy; NRS-2002.

2 Servicio de Nutrición Clínica, Hospital General de México. 


\section{INTRODUCCIÓN}

La nutrición clínica ha tenido un desarrollo constante desde sus primeros inicios con la nutrición artificial en el siglo XIX y principios del XX, donde personajes como Claude Bernard comenzaron los experimentos que posteriormente, en los años setenta, verían resultados como los demostrados por el Dr. Stanley J. Dudrick ${ }^{(1,2)}$.

De la década de los ochenta a la actualidad el conocimiento obtenido en nutrición clínica ha tenido mayor relevancia. La asociación entre la desnutrición y los desenlaces médicos y complicaciones en diversas patologías ha creado la necesidad imperiosa de herramientas de tamizaje que ayuden a identificar los pacientes en riesgo de desnutrición y se beneficien de la terapia nutricional. Desde el año 2002 se ha implementado en varios países la herramienta de tamizaje para el riesgo nutricional hospitalario Nutritional Risk Screening (NRS - 2002), recomendada por la European Society for Clinical Nutrition and Metabolism (ESPEN) ${ }^{(3,4)}$. En estudios europeos esta herramienta ha demostrado sensibilidad, que va de $62 \%$ - $93 \%$ y una especificidad de entre $80 \%$ y 93,1\%, además de ser un adecuado predictor de complicaciones ${ }^{(5-7)}$.

A pesar del trabajo interdisciplinario de la nutrición con los servicios que abarcan las diferentes especialidades médicas, existe un amplio desconocimiento por parte del personal tratante acerca de la importancia, tanto de identificar el riesgo nutricional, como de diagnosticar la desnutrición durante la hospitalización de los pacientes ${ }^{(8)}$. Se ha reportado que menos de $20 \%$ de los expedientes clínicos contienen información relacionada con el estado nutricional en Latinoamérica ${ }^{(9)}$. Además, no se ha estudiado la relación entre el riesgo nutricional y la solicitud de valoración nutricional en los distintos servicios hospitalarios. Este punto es importante ya que el tamizaje es el primer paso del cuidado nutricional el cual conlleva a una valoración completa y posterior intervención nutricional. El objetivo del estudio fue comparar la proporción de interconsultas realizadas al servicio de nutrición clínica con el riesgo nutricional en los distintos servicios clínicos del Hospital General de México.

\section{METODOLOGÍA}

Se realizó un estudio observacional, analítico, transversal y retrospectivo en los servicios del Hospital General de México. Se incluyeron todos los pacientes adultos que ingresaron al área de hospitalización de 17 servi- cios durante el periodo de enero 1 a 31 de diciembre de 2017.

Se consultaron las historias clínicas de todos los pacientes adultos para obtener el resultado del tamizaje nutricional realizado con la herramienta NRS-2002 ${ }^{(3)}$. Se excluyeron los pacientes que estuvieron hospitalizados únicamente en el servicio de urgencias. En el Hospital de México el tamizaje se realiza como parte de la valoración rutinaria inicial en las primeras 24 - 48 horas del ingreso al servicio clínico hospitalario.

La NRS - 2002 consta de dos etapas: un tamizaje inicial y una valoración del riesgo nutricional, esta última está basada en un puntaje el estado nutricional y severidad de la enfermedad. El puntaje va del cero al tres, donde cero es indicador de estado nutricional normal y tres o más, de riesgo nutricional.

Se obtuvo el porcentaje de interconsultas realizadas por parte del personal médico al servicio de nutrición clínica a partir de los registros mensuales digitales de interconsultas. Se contemplaron aquellas de primera vez en la población total según el servicio hospitalario, incluidas aquellas que fueron solicitadas para pacientes que no presentaron riesgo nutricional a su ingreso. Se compararon por cada servicio clínico, el número de interconsultas por paciente y el riesgo nutricional.

El análisis de los datos se efectuó por medio de estadística descriptiva en forma de proporciones para los pacientes con riesgo nutricional y se comparó con el número de interconsultas médicas realizadas, por medio del programa STATA v.14. Los resultados se expresan en forma de porcentajes considerando un intervalo de confianza de $95 \%$.

\section{RESULTADOS}

Durante 2017 se registraron 38.960 pacientes y se evaluaron 31.225 pacientes, de los cuales $19,20 \%$ ( $n=$ 5.996 pacientes) fueron clasificados con riesgo nutricional. A 16,1\% de los pacientes en los servicios clínicos no se realizó tamizaje nutricional (Figura 1).

De los pacientes con riesgo nutricional $(\mathrm{n}=5.996)$, $81,42 \%(n=4.880)$ tuvieron interconsultas al servicio de nutrición clínica. Los servicios con mayor proporción de riesgo nutricional fueron nefrología 95,26\% (IC $95 \%$ ), seguido de geriatría 66,46 \% (IC $95 \%$ ), neurología y neurocirugía $68,31 \%$ (IC $95 \%$ ), oncología 63,23 \% (IC 95\%) e Infectología 47,71 \% (IC $95 \%$ ) (Tabla 1).

Por otro lado, algunos servicios sobrepasaron las interconsultas realizadas en relación con los pacientes detectados con riesgo, como lo fueron ginecología y obs- 


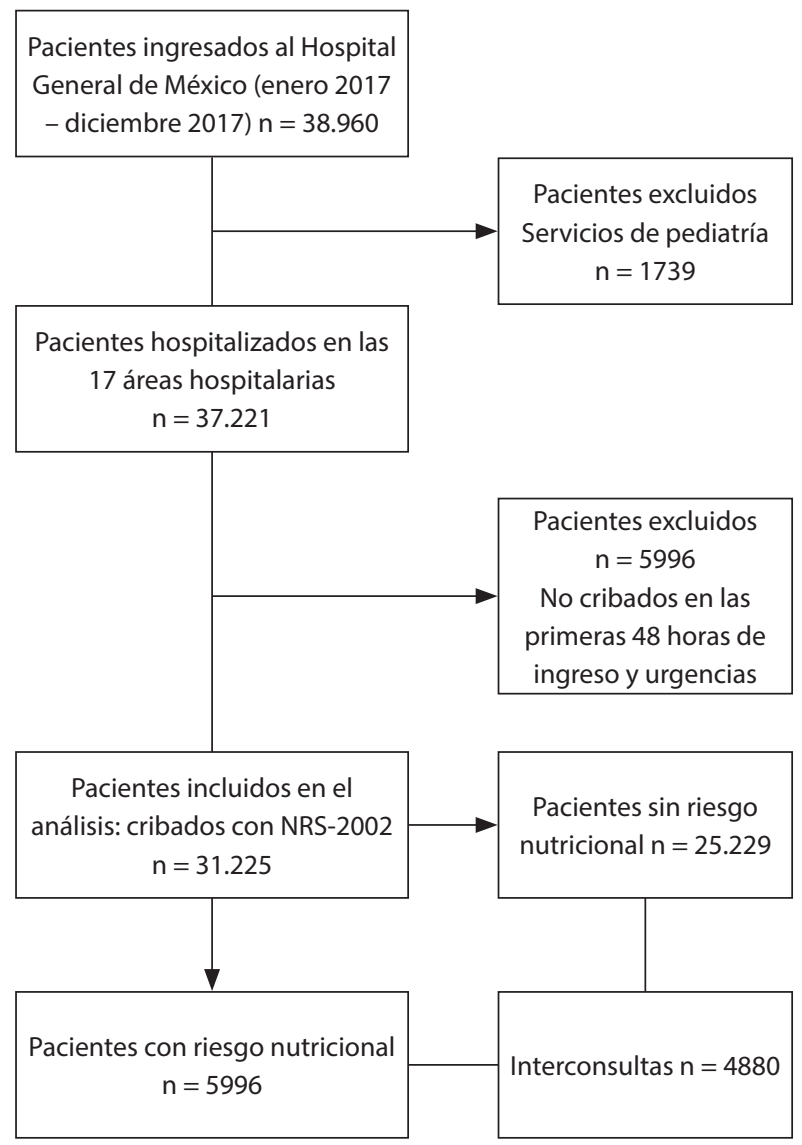

Figura 1. Diagrama de flujo del estudio

tetricia $338,2 \%$, cirugía 331,8 y oncología $143,5 \%$. Los servicios con menor porcentaje de interconsultas, a pesar del riesgo detectado para el servicio de nutrición clínica, fueron: oftalmología 1,19\% de interconsultas (IC $95 \%$ ), urología 12,83 \% (IC $95 \%$ ), nefrología 13,32 \% (IC 95 $\%)$ y geriatría $16,27 \%$ (IC $95 \%$ ), el resto de los resultados se encuentran desglosados en la Tabla 1.

\section{DISCUSIÓN}

El presente estudio mostró, según la herramienta NRS 2002, que el 19,20\% de los pacientes en el Hospital General de México presentaron riesgo nutricional. En Latinoamérica, la prevalencia de riesgo nutricional varía según la población estudiada y el tipo de herramienta utilizada ${ }^{(9)}$. Cifras similares a las nuestras y empleando la misma herramienta, fueron encontradas en población general por Raslan, et $\mathrm{al}^{(10)}$, y Cardinal, et al ${ }^{(11)} \operatorname{con} 27,9$ $\%$ y $20 \%$ respectivamente, así como los observados por Meireles, et al de 19,3\% en pacientes quirúrgicos ${ }^{(12)}$.
Llama la atención que nuestros resultados mostraron un alto porcentaje de riesgo nutricional en los pacientes del servicio de nefrología 95,26\% ( $\mathrm{n}=503)$. Esto difiere del estudio de Borek, et al ${ }^{(13)}$, el cual mostró que, utilizando la misma herramienta, 40 \% de 292 pacientes admitidos de manera consecutiva durante seis meses al servicio de nefrología de un hospital universitario presentaron riesgo de desnutrición.

En este estudio no se estableció diferencia entre los pacientes que reingresaron al hospital. La población del servicio de nefrología ingresa al hospital en forma recurrente al tratamiento dialítico, lo cual puede influir en la prevalencia de riesgo encontrada. Existe evidencia de que la población nefrópata tiene un alto riesgo de desnutrición ${ }^{(13)}$ relacionado con condiciones hipermetabólicas e infecciosas ${ }^{(14-17)}$.

En el caso del Servicio de Oncología, 63,23 \% de los pacientes tuvieron riesgo nutricional. Esta cifra es superior a la prevalencia del 50,2\% encontrada en el año 2014 en la misma población y herramienta de tamizaje ${ }^{(18)}$.

En la población con enfermedades neurológicas se ha visto que el porcentaje de riesgo nutricional asciende hasta $46 \%{ }^{(17)}$, esto es $20 \%$ menos de lo observado en nuestros resultados. Otra población vulnerable son los pacientes geriátricos, que debido al proceso de envejecimiento y otros factores asociados, presentan pérdida de masa muscular y mayor riesgo de desnutrición. El presente estudio mostró que $66 \%$ de los pacientes del servicio de geriatría tenía riesgo nutricional, cifra comparable con lo reportado en la literatura ${ }^{(19)}$.

El aspecto administrativo es la razón principal por la cual a $16,1 \%$ de los pacientes en los servicios clínicos no se les realizara tamizaje nutricional.

Los resultados observados en nuestro estudio muestran que algunos de los servicios con mayor riesgo nutricional presentan una baja proporción de interconsultas como es el caso de los servicios de nefrología y de geriatría, que pese a ser poblaciones de alto riesgo nutricional, registran un bajo número de solicitudes de valoración nutricional por parte del personal médico.

En este estudio no se clasificaron los pacientes según patología específica dado que no fue objetivo propuesto. Por ejemplo, en servicios como el de medicina interna, hay una población importante de pacientes diabéticos con enfermedad renal crónica sometidos a diálisis peritoneal, sin ser de manera exclusiva derivados al área de nefrología.

A pesar de que se solicitó interconsulta en 81,42\% de los pacientes en riesgo nutricional, esto no fue equitativo en todos los servicios. $\mathrm{Al}$ analizar el total de interconsul- 
Tabla 1. Interconsultas según el riesgo nutricional por servicio

\begin{tabular}{|c|c|c|c|c|c|c|}
\hline Servicio & n cribados & $\mathrm{n}$ en riesgo & Riesgo (\%) & IC $95 \%$ & $\begin{array}{c}\text { Interconsultas } \\
(\%)\end{array}$ & IC $95 \%$ \\
\hline Oftalmología & 1.682 & 84 & 4,99 & $(4,02-6,14)$ & 1,19 & $(0,030-6,44)$ \\
\hline Urología & 2.539 & 709 & 27,92 & $(26,18-29,71)$ & 12,83 & $(10,46-15,52)$ \\
\hline Nefrología & 528 & 503 & 95,26 & $(93,08-96,91)$ & 13,32 & $(10,47-16,60)$ \\
\hline Geriatría & 647 & 430 & 66,46 & $(62,67-70,09)$ & 16,27 & $(12,91-20,11)$ \\
\hline Otorrinolaringología & 1.692 & 252 & 14,89 & $(13,22-16,68)$ & 24,20 & $(19,05-29,97)$ \\
\hline $\begin{array}{l}\text { Endocrinología- } \\
\text { Reumatología }\end{array}$ & 686 & 57 & 10,53 & $(6,35-10,63)$ & 29,82 & $(18,42-43,40)$ \\
\hline Cardiología & 1.405 & 105 & 7,47 & $(6,15-8,97)$ & 47,61 & $(37,78-57,59)$ \\
\hline Neumología & 879 & 165 & 18,77 & $(16,24-21,51)$ & 51,51 & $(43,61-59,35)$ \\
\hline $\begin{array}{l}\text { Neurología y } \\
\text { Neurocirugía }\end{array}$ & 445 & 304 & 68,31 & $(63,76-72,61)$ & 64,80 & $(59,14-70,16)$ \\
\hline Medicina Interna & 4.014 & 1.238 & 30,84 & $(29,41-32,29)$ & 69,06 & $(66,40-71,63)$ \\
\hline Gastroenterología & 1.618 & 295 & 18,23 & $(16,37-20,20)$ & 77,28 & $(72,07-81,94)$ \\
\hline Dermatología & 694 & 40 & 5,76 & $(4,14-7,76)$ & 77,50 & $(61,54-89,16)$ \\
\hline Ortopedia & 1.984 & 40 & 2,01 & $(1,44-2,73)$ & 102,50 & * \\
\hline Infectología & 415 & 198 & 47,71 & $(42,81-52,63)$ & 119,19 & * \\
\hline Oncología & 1.991 & 1.259 & 63,23 & $(61,07-65,35)$ & 143,53 & * \\
\hline Cirugía & 5.768 & 245 & 4,24 & $(3,74-4,80)$ & 331,84 & * \\
\hline $\begin{array}{l}\text { Ginecología y } \\
\text { Obstetricia }\end{array}$ & 4.238 & 68 & 1,60 & $(1,24-2,02)$ & 338,24 & * \\
\hline
\end{tabular}

* Los valores exceden la proporción

$\mathrm{n}=$ población estudiada, $\%=$ porcentaje, $\mathrm{IC}=$ intervalo de confianza

tas, es importante resaltar que en algunos casos se solicitaron para pacientes que no tenían riesgo nutricional al ingreso, como lo fueron ginecología y obstetricia, cirugía, oncología, infectología y ortopedia. En otros servicios pese al riesgo nutricional detectado en el cribado inicial, el médico tratante no solicitó la interconsulta.

Algunos estudios muestran que hasta $70 \%$ del personal médico carece de conocimientos suficientes en el área de nutrición clínica para solicitar este apoyo en forma oportuna ${ }^{(20-22)}$. La actitud y conocimientos sobre el riesgo nutricional y la utilidad de solicitar interconsulta al servicio de nutrición merece ser evaluado en nuestra institución.

Es necesario reforzar el trabajo conjunto en los servicios hospitalarios ya que la desnutrición es una de las principales complicaciones que ocurren durante la estancia hospitalaria por lo que el tratamiento oportuno puede brindar al paciente mejor pronóstico y menor riesgo de complicaciones. Es importante hacer énfasis en pacientes en las áreas de mayor riesgo nutricional y realizar el seguimiento durante su estancia.

\section{CONCLUSIONES}

Este estudio muestra que el porcentaje de solicitud de interconsulta al servicio de nutrición clínica por parte del médico tratante no se relaciona con el porcentaje de riesgo nutricional del servicio. El riesgo nutricional varía de manera importante según el servicio hospitalario. Es necesario reforzar el trabajo conjunto en los servicios hospitalarios y dar seguimiento a los pacientes con riesgo nutricional de manera oportuna.- 


\section{Referencias bibliográficas}

1. Bernabeu Mestre J, Ballester Añón R, Lozano CWB, López ÁF, Culebras JM, Sanz Valero J, et al. Historia de la nutrición clínica española (1): Nutrición artificial y su incorporación al ámbito clínico español. Nutr Hosp. 2015;32(5):1843-52.

2. Dudrick SJ, Wilmore DW, Vars HM, Rhoads JE. Long-term total parenteral nutrition with growth, development, and positive nitrogen balance. Surgery. 1968;64(1):134-42.

3. Kondrup J, Ramussen HH, Hamberg O, Stanga Z, Camilo M, Richardson R, et al. Nutritional risk screening (NRS 2002): A new method based on an analysis of controlled clinical trials. Clin Nutr. 2003;22(3):321-36.

4. Kondrup J, Allison SP, Elia M, Vellas B, Plauth M. ESPEN guidelines for nutrition screening 2002. Clin Nutr. 2003;22(4):415-21.

5. Gómez-Candela C, Serrano Labajos R, García-Vazquez N, Valero Pérez M, Morato Martínez M, Santurino Fontecha C, et al. Proceso completo de implantación de un sistema de cribado de riesgo nutricional en el hospital universitario La Paz de Madrid. Nutr Hosp. 2013;28(6):2165-74.

6. Neelemaat F, Meijers J, Kruizenga H, Van Ballegooijen H, Van Bokhorst-de van der Schueren M. Comparison of five malnutrition screening tools in one hospital inpatient sample. J Clin Nurs. 2011;20(15-16):2144-52.

7. Kyle UG, Kossovsky MP, Karsegard VL, Pichard C. Comparison of tools for nutritional assessment and screening at hospital admission: A population study. Clin Nutr. 2006;25(3):409-17.

8. Goiburu Bianco ME, Alfonzo LF, Aranda AL, Riveros MF, Ughelli MA, Dallman D, et al. Nivel de conocimiento en nutrición clínica en miembros del Equipo de Salud de Hospitales Universitarios del Paraguay. Nutr Hosp. 2006;21(5):591-5.

9. Correia MITD, Perman MI, Waitzberg DL. Hospital malnutrition in Latin America: A systematic review. Clin Nutr. 2017;36(4):958-67.

10. Raslan M, Gonzalez MC, Dias MCG, Nascimento M, Castro $\mathrm{M}$, Marques $\mathrm{P}$, et al. Comparison of nutritional risk screening tools for predicting clinical outcomes in hospitalized patients. Nutrition. 26(7-8):721-6.

11. Cardinal TR, Wazlawik E, Bastos JL, Nakazora LM, Scheunemann L. Standardized phase angle indicates nutri- tional status in hospitalized preoperative patients. Nutr Res. 2010;30(9):594-600.

12. Meireles MS, Wazlawik E, Bastos JL, Garcia MF. Comparison between nutritional risk tools and parameters derived from bioelectrical impedance analysis with subjective global assessment. J Acad Nutr Diet. 2012;112(10):1543-9.

13. Borek P, Chmielewski M, Małgorzewicz S, Dębska Ślizień A. Analysis of Outcomes of the NRS 2002 in Patients Hospitalized in Nephrology Wards. Nutrients. 2017;9(3):287.

14. Argilés JM. Cancer-associated malnutrition. Eur J Oncol Nurs. 2005;9(Suppl 2):S39-50.

15. Gupta D, Vashi PG, Lammersfeld CA, Braun DP. Role of nutritional status in predicting the length of stay in cancer: a systematic review of the epidemiological literature. Ann Nutr Metab. 2011;59(2-4):96-106.

16. Kyle UG, Genton L, Pichard C. Hospital length of stay and nutritional status. Curr Opin Clin Nutr Metab Care. 2005;8(4):397-402.

17. Sorensen J, Kondrup J, Prokopowicz J, Schiesser M, Krähenbühl L, Meier R, et al. EuroOOPS: an international, multicentre study to implement nutritional risk screening and evaluate clinical outcome. Clin Nutr. 2008;27(3):340-9.

18. Alvarez-Altamirano $\mathrm{K}$, Delgadillo $\mathrm{T}$, García-García A, Alatriste-Ortiz G, Vanessa FT. Prevalencia de riesgo de desnutrición evaluada con NRS-2002 en población oncológica mexicana. Nutr Hosp. 2014;30(1):173-8.

19. Agarwal E, Miller M, Yaxley A, Isenring E. Malnutrition in the elderly: a narrative review. Maturitas. 2013;76(4):296-302.

20. Grammatikopoulou MG, Katsouda A, Lekka K, Tsantekidis $\mathrm{K}$, Bouras E, Kasapidou E, et al. Is continuing medical education sufficient? Assessing the clinical nutrition knowledge of medical doctors. Nutrition.2018; In press.

21. Paulo DA, de Oliveira BMR, Wang DWM, Guimaraes MP, Cukier C, Lopes Filho G de J. Surgeons' knowledge and attitude regarding concepts of nutritional therapy. Rev Col Bras Cir. 2013;40(5):409-19.

22. Abdollahi M, Houshiarrad A, Abtahi M, Esmaeli M, Pouraram $\mathrm{H}$, Khoshfetrat MR, et al. The nutrition knowledge level of physicians, nurses and nutritionists in some educational hospitals. J Paramed Sci. 2013;4(1):106-14. 